\title{
On Calculation of Fractal Dimension of Color Images
}

\author{
Soumya Ranjan Nayak \\ College of Engineering and Technology Department of Information Technology Bhubaneswar, 751003, India \\ Email: nayak.soumya17@gmail.com \\ Jibitesh Mishra \\ College of Engineering and Technology Department of Computer Science and Application, Bhubaneswar, 751003, \\ India \\ Email: jmishra@cet.edu.in
}

\begin{abstract}
Fractal Dimension is a basic parameter of fractal geometry and it has been applied in many fields of application including image analysis, texture segmentation, and shape classification. Many fractal dimensions methods have been evolved depending upon different types of images that could be differentiated with greater precision. In this paper, we propose a color approach based on the modified differential box-counting method to estimate fractal dimension of color images in terms of its smoothness. Here we have experimented on four sets of color images like; sixteen number of real natural texture images, eight sets of controlled experimental fabric images with varied color and texture, twelve numbers of generated synthetic images and four smoothed images of known fractal dimension. The results demonstrated that the said proposed method shows accurate fractal dimension estimation of color texture image and also it indicates FD as 2 for smoothed images, which has already been developed in last decade and indicates higher roughness in color images, to check the accuracy of our proposed method, we used a set of twelve synthetic generated images.
\end{abstract}

Index Terms-Fractal, (IDBC), Color images, Box Counting, Roughness.

\section{INTRODUCTION}

The fractal geometry is a concept which was firstly invented by Mandelbrot for express self-similar sets of fractals images like mountain and cloud which are unfeasible to illustrate by means of classical geometry [1] The concept Fractal geometry is broadly used in many application of image processing in terms of the precise evaluation of dimension of complex and irritated objects. These concepts are primarily evolved to decrease the differences of surface roughness among the artificial designs and natural scenes. Since artificial designs typically have a plain and simple texture surface and measure geometry shape, from this point of view, this concept are easily used in various recognition of pattern applications such as categorization and segmentation and many more. The major parts of fractal geometry are called self-similarity. The theory of self-similarity should be defined as the object is precisely or something like related to a part of itself; alternatively we can say that if a surrounded set A is supposed to be self-similar, if A is the unification of a number $(\mathrm{Nr})$ of non-overlapping scaled copies of itself, where $r$ is the reduction factor reported in [1]. Fractal geometry also gives a mathematical special model of various difficult objects initiate in nature [1-3], that includes coastline, mountains, and clouds and also it has been useful in different texture analysis such as segmentation [4-6], shape measurement and classification [7], image and graphic analysis in different fields [8, 9]. In this regard, different researchers are contributed their work, such as Gangepain and Roques-carmes presented reticular cell counting method [10,11] and later on Keller et al. proposed probability box counting [10,12]. Both Gangepain and Keller gives the satisfactory result, but after reaching a particular level of image intensity surface then the slope of the arc tends to zero; therefore both the methods does not estimate the accurate FD. In order to avoid such situation, Sarkar et. al. presented a novel technique called differential box-counting (DBC) technique [4, 10, 13], this DBC method treated as the efficient one found in many articles like[14,15]. However in the case of DBC method there are three major problems reported by Jain et. al. [16]. Which are box height selection, calculation of the box number and partition of the image intensity surface? In order to avoid these situations, the same author presented another method called an improved box-counting method [16] in order to avoid the three above mention drawbacks of DBC method. Again some demerits found in DBC method reported by Chen.et.al [17] that is over-counting and under-counting. To avoid such situation the same author [17] presented another method called improve DBC. The box-counting algorithms are often used for estimation of surface roughness due to its easiness reported in [3].

A reminder of this article is as follows; Section 2 presented the essential ideas about fractal dimension and FD estimation of color images. Section 3 discusses proposed methodology. Section 4 describes experimental 
result and discussion and concluding remark are presented in section 5 .

\section{BASIC FRACTAL DIMENSION}

A set can be defined as a fractal, for which the Hausdorff Besikovich dimension is firmly bigger than the topological dimension. The estimation of fractal dimension is a necessary feature of fractal for the cause that it has got information concerning their geometric structure. The fractal dimension of the entire images generally distribution of intensity values, more deliberately, estimating the roughness of an entire image. The basic theory to estimate roughness of a whole image depends on the theory of self-similarity. From the property of self-similarity we can say that the fractal objects are normally an irregular or inexact geometric shape, if we assume the fractal object is the combination of $\mathrm{N}$ distinct of non-overlapping copies of itself, each of which is similar to the original and the ratio factor are scaled down or up by $r$, then the fractal dimension $\mathrm{D}$ is given by equation (1).

$$
D=\frac{\log (N)}{\log (1 / r)}
$$

\section{A. Improved Differential Box-Counting}

Liu.et.al [18] suggested improved DBC method for eliminating the demerits found on original DBC method that is over counting and under counting. In this regard to implement their method, they taking into consideration by which maximum and minimum intensity value falls in grids of the block. First of all, they taking an image of size $\mathrm{M} \times \mathrm{M}$ in $3 \mathrm{D}$ space, where the coordinate of $(\mathrm{x}, \mathrm{y})$ and coordinate $(\mathrm{z})$ represents the $2 \mathrm{D}$ position and gray level. Then they scaled down the total no of pixels into box size of $1 \times 1$ where 1 is lies between 1 to $M / 2$ and estimate $r=1$ /M. On each box of the block, there is a pillar of boxes of total size is s x s x s', and s' represents the height of each box and it can be calculated as $\mathrm{G} / \mathrm{s}^{\prime}=\mathrm{M} / \mathrm{s}$, where $\mathrm{G}$ represents the total gray-levels. The minimum and maximum gray levels are represented as $I_{\min }$ and $I_{\max }$ respectively in the $(i, j)$ th block. Then the total number of boxes needed to cover the block in the $\mathrm{z}$ direction is $\mathrm{nr}$ old and after shifting the $\delta$ positions from $\mathrm{nr}_{\text {old }}, \mathrm{nr}_{\text {new }}$ is calculated. Maximum of $\mathrm{nr}_{\text {old }}$ and $\mathrm{nr}_{\text {new }}$ is taken as $\mathrm{nr}$. $\mathrm{nr}=\max \left(\mathrm{nr}_{\text {old }}, \mathrm{nr}_{\text {new }}\right) . \mathrm{nr}_{\text {old }} \mathrm{or}_{\mathrm{nr}} \mathrm{r}_{\text {new }}$ is calculated as follow:

$$
n_{r}(i, j)=c e i l\left\{\begin{array}{l}
\left(\frac{I_{\max }-I_{\min }+1}{s^{\prime}}\right) i f \ldots I_{\max } \neq I_{\min } \\
1 \ldots \ldots \ldots \ldots \ldots \ldots \ldots \ldots \ldots \ldots \ldots . . . \text { Otherwise }
\end{array}\right.
$$

And $\mathrm{Nr}$ will be calculated as

$$
N_{r}=\sum n_{r}(i, j)
$$

The fractal dimension (IDBC) is calculated with regression plot between $\log (\mathrm{N})$ vs $\log (1 / \mathrm{r})$.

There are many algorithms were proposed in terms of gray scale and color approach. Maximum of research are done using grayscale image like reticular cell counting method [11], kellers approach [12], Differential box counting method [13], Improved differential boxcounting method [17] and only two research papers have been evolved so far in case of color images like color version of probability method [19] is the modified version of Keller approach and box merging method [20] is the extended version of box counting mechanism. However In this article we have considering improved differential box-counting method which has already been developed for estimating fractal dimension of gray scale image and added our proposed methodology to efficiently estimate the fractal dimension of color image and compared with color version of box merging method with different experimental analysis in terms of different class of color texture images and known fractal dimension images and shows the time complexity of both algorithm.

\section{B. Fractal Dimension Estimation of Color Images}

Nowadays the world of images became colored. Digital color images are related to grayscale images and the pixels are arranged in a grid of boxes. However, in the case of color images, each individual pixel has three sampled value mainly red, green and blue. Therefore estimating fractal dimension of color images is a very challenging issue. Therefore in this regard, extremely limited approaches that connect to calculate the roughness of color images and the principle is only constrain to trivial color investigation and convert a gray scale problem into a false color one [20].

\section{Proposed Methodology}

The color estimation proposed by Ivanovici and Richard [19] called probabilistic algorithm is given by Voss and another method developed by Nikolaidis et al. [20] called box merging method based on the concept of box counting mechanism and Zhao and Wang [21] presented another box counting based on the concept of maximum color distance to estimate roughness of color images based on the 5-D vector. In order to validate their both methods, Ivanovici and Richard [19] generate color fractal images based on Hurst parameter by using the technique of the midpoint displacement algorithm and Nikolaidis et al. [20] used known fractal dimension image and checked for validation. However, we are proposing improved differential box-counting method based on the same 5-D vector to estimate roughness of color images using the RGB components of the color image, which roughness is lies between 2 to 5 . Therefore in order to estimate roughness of color image, we first estimate roughness of each individual RGB component and subtracted its corresponding smoothness. After subtraction of smoothness, we will take its fractional part and add its corresponding RGB components and finally the addition of smoothness to get accurate roughness. 


$$
\begin{aligned}
& D_{R}=D_{I D B C(R)}-2 \\
& D_{G}=D_{I D B C(G)}-2 \\
& D_{B}=D_{I D B C(B)}-2 \\
& D_{\text {Color }}=2+D_{R}+D_{G}+D_{B}
\end{aligned}
$$

\section{A. Algorithm Summery}

The detailed algorithm can be summarized as follows:

- Picking the different grid box sizes of 2 up to $M / 2$ to wrap the whole image.

- Take into account the number of the total boxes require to cover the image entirely.

- Using the slope of least-squares linear fit of $\log$ (Nr) vs $\log (1 / r)$ to achieve the FD of each red, green and blue channels.

- After calculating FD of each individual channel, we subtracted its corresponding smoothness and take its fractional part and add its corresponding RGB components and finally addition of smoothness to get accurate roughness.

\section{EXPERIMENTAL ANALYSIS}

In this study, our proposed method is implemented on matlab14 in windows 864 bit operating system, Intel (R) i7 - 4770 CPU @ $3.40 \mathrm{GHz}$. Our proposed method and box merging method have been chosen for our four sets of images for this experimental analysis, shown in Figs. 1 to 4 , and one set of 16 color textured images downloaded from Brodatz database [18], shown in "Fig. 1,". One set of 8 own created textile fabric of exactly similar texture with varied color were collected for this ground truth experiment shown in "Fig. 2,". The images were taken in color viewing cabinet with D65 illuminant, viewed at 45degree angle with white balance in manual mode. As the last test, we are taking four set of smooth known fractal

\begin{tabular}{|c|c|c|}
\hline & Start & \\
\hline & Read image & $\%$ input the image \\
\hline Step (1) & For $\mathrm{i}=1: 3$ & \\
\hline & $\operatorname{Img}(\mathrm{i})=(:,:, \mathrm{i})$ & \\
\hline & {$[\mathrm{p}, \mathrm{q}]=$ size of $(\mathrm{img}(\mathrm{i}))$} & $\%$ read the sizes of the image \\
\hline & Select exact grid box size $\mathrm{g}(\mathrm{b})$; & \\
\hline & $\mathrm{B}=1$ & $\%$ using $\mathrm{B}$ as a variable of different grid box sizes \\
\hline Step (2) & While $(\mathrm{g}(\mathrm{b})<\mathrm{p} / 2)$ & \\
\hline & $\mathrm{r}=\mathrm{g}(\mathrm{b}) / \mathrm{p}$ & $\%$ define $r$ \\
\hline Step (3) & For $\mathrm{i}<\mathrm{p} / \mathrm{g}(\mathrm{b}), \mathrm{j}<\mathrm{q} / \mathrm{g}(\mathrm{b})$ & \\
\hline & Count $\mathrm{n}_{\mathrm{r}-\mathrm{old}}$ & $\%$ use (2) \\
\hline & Move the box block in $(\mathrm{x}, \mathrm{y})$ plane within t pixels; & $\%$ under the set rule \\
\hline & Count $\mathrm{n}_{\mathrm{r}-\mathrm{new}}$; & $\%$ use $(2)$ \\
\hline & $\begin{array}{l}\mathrm{n}_{\mathrm{r}}=\max \left(\mathrm{n}_{\mathrm{r}-\mathrm{old}}, \mathrm{n}_{\mathrm{r}-\mathrm{new}}\right) ; \\
\text { End for }\end{array}$ & \\
\hline & $\mathrm{N}_{\mathrm{r}}=\operatorname{sum}\left(\mathrm{n}_{\mathrm{r}}\right)$ & $\%$ calculate the total number of boxes \\
\hline & $\mathrm{B}=\mathrm{b}+1$ & $\%$ for using dissimilar grid box sizes \\
\hline & End while & \\
\hline & Call fit $\left(\log \mathrm{N}_{\mathrm{r}}, \log (1 / \mathrm{r})\right)$; & $\%$ execute least-squares linear fit \\
\hline & Obtain FD of img(i); & equal to the slope of the fitted line of each channel \\
\hline Step (4) & $D_{\text {color }}=2+($ FD of img(i) -2$)$ & \%Color FD estimation using $(4)$ \\
\hline & End for & \\
\hline & End & \\
\hline
\end{tabular}
dimension image which fractal dimension was measured as 2 , shown in Table 3.

Our details procedure is listed in Table below

\section{A. Result and Discussion}

Using the improved differential box-counting method to estimate the fractal dimension of color images in terms of its smoothness, for the same we are estimating the FD of color images using the individual red, green and components of the input color image based on the same 5 -D vector, which roughness is lies between 2 to 5 . Therefore in order to estimate roughness of color image, we first estimate roughness of each individual RGB component and subtracted its corresponding smoothness. After subtraction of smoothness, we will take its fractional part and add its corresponding RGB components and finally the addition of smoothness to get accurate roughness of color images. For our experimental work, we took three different class of texture images, first of all for our first experimental analysis we have to consider different texture taken from Brodatz's image database [18] given in "Fig. 1," and it shows that D93 image has got more color roughness as per both box 
merging and our proposed method, also from Table. 1, it is clear that those texture images having more color roughness as estimated in box merging method are also having the higher roughness in our proposed method. However, it seems from Table. 1 that method proposed by us is able to compute higher color roughness based on the RGB components of the color texture. For our second experimental analysis, we are considering our own created eight textile fabric of exactly similar texture with varied color were collected for this ground truth experiment shown in "Fig. 2,". The images were taken in color viewing cabinet with D65 illuminant, viewed at 45degree angle with white balance in manual mode and the camera specification are listed below. It is clear from the Table 2, that image I2 is having least fractal Dimension and I3 is having highest Fractal dimension among the eight color texture images, this can be easily established by the fact that under natural human perception I 2 seems to be having more smoothness and I3 maximum roughness, this has also been validated using box merging method. In last experimental analysis is used for validation purpose, in this case, we are considering four
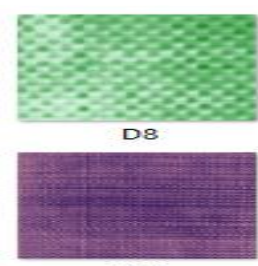

D55
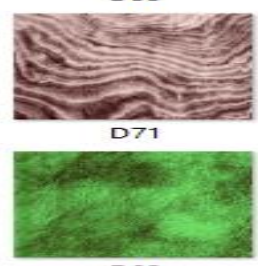

D93
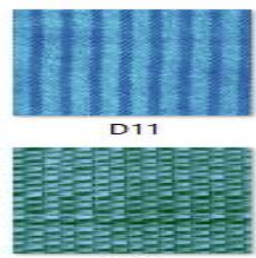

D56
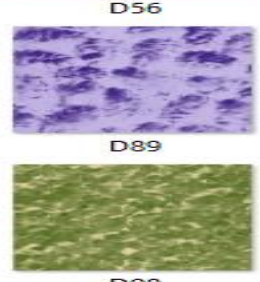

smooth images shown in "Fig. 3,". All the articles on fractal dimension mention that fractal dimension of the smooth image as accepted as 2.0, also in our case Table 3 indicates that all four images having fractal dimension as 2 , hence it is crystal clear that our method is more robust to estimate the fractal dimension of color images.

\section{B. Experiment on Real Natural Texture Images}

In this section, we are using a set of 16 real texture images [18] from the brodatz database for our experimental analysis, which is represented in "Fig. 1," Their corresponding FD are listed in Table 1. The FD generated from proposed method are in the range from 2.6503 to 4.1718 , similarly, the other measure like box merging method are range from 2.4829 to 2.5868 and 3.5155 to 2.5821 respectively, and the detail graph is presented in "Fig. 2," from this result it is crystal clear that our proposed method efficiently capture the exact roughness of color images as compared to box merging method.
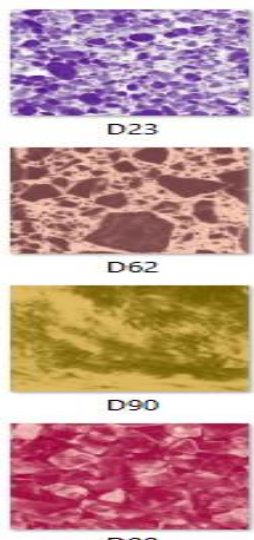

099
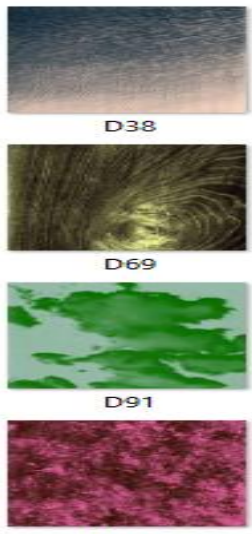

0100

Fig.1. Sixteen real texture images.

Table 1. Fractal Dimension of Sixteen Real Texture Images

\begin{tabular}{|c|c|c|}
\hline $\begin{array}{c}\text { Image } \\
\text { Name }\end{array}$ & Proposed & Box Merging \\
\hline D8 & 3.1268 & 2.9327 \\
\hline D11 & 3.9343 & 3.3754 \\
\hline D23 & 3.5673 & 3.5070 \\
\hline D38 & 3.7363 & 2.8334 \\
\hline D55 & 4.0583 & 3.4889 \\
\hline D62 & 3.1462 & 3.3308 \\
\hline D56 & 3.5793 & 2.8243 \\
\hline D69 & 3.7475 & 3.0567 \\
\hline D71 & 3.8522 & 3.0087 \\
\hline D89 & 3.2513 & 2.9213 \\
\hline D90 & 3.0201 & 2.6285 \\
\hline D91 & 2.6503 & 2.4829 \\
\hline D93 & 4.1718 & 3.5155 \\
\hline D98 & 3.0779 & 2.8149 \\
\hline D99 & 3.1934 & 3.0304 \\
\hline D100 & 3.8735 & 3.5007 \\
\hline & & \\
\hline
\end{tabular}




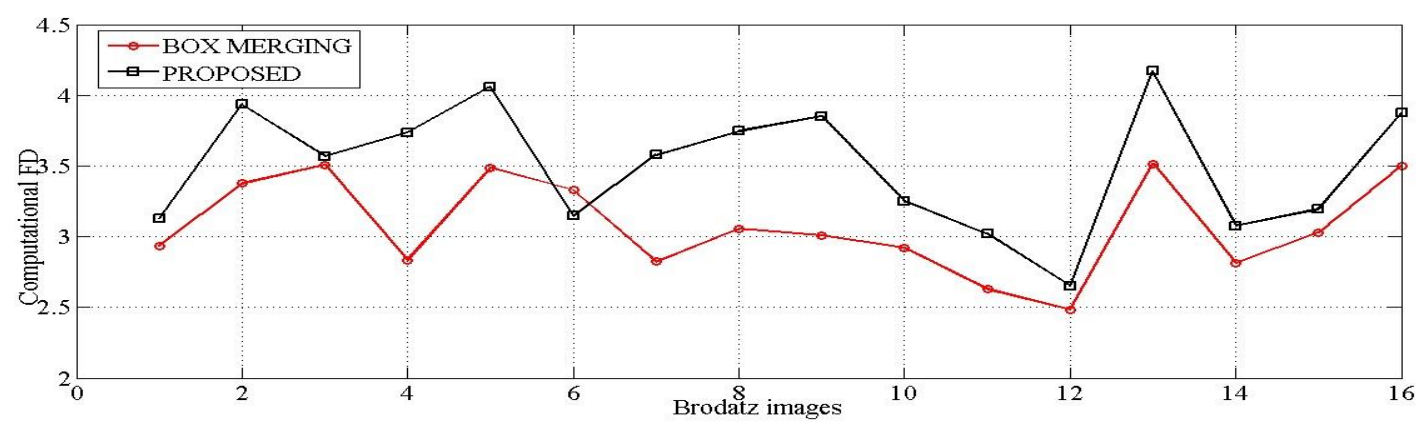

Fig.2. Computational FD of sixteen real texture images.

\section{Experiment on Own Created Texture Images}

In this section, we are using a set of 8 of controlled experimental fabric images with varied color and texture, which are represented in "Fig. 3," Their corresponding FD are listed on Table 2, from the visually inspection we have seen that image $\mathrm{I} 2$ is more smoother than other images, as it is smoother then FD should be less than other images as mention on Table 2, our proposed method provides FD greater than 3 except I 2 images but in the case of box merging method, the only I3 image provides FD as greater than 3 and all other images having FD between 2 to 3 . From this point, it is crystal clear that our proposed method efficiently captures the exact roughness of color images as compared to box merging method.

The specification of camera and imaging are as follows for our own created texture image shown in figure. 2

Camera model:

F-stop:

Exposure time:

ISO speed: 2000

Exposure bias :

Focal length :

Max Aparature :

Aperture mode :

Tv(Shutter Speed)

Av(Aperture Value)

Metering Modes Center

Exposure Compensation

ISO Speed

Lens Focal Length

Image size

White Balance

Parameters

Sharpness level :

Contrast :

Sharpness :

Color saturation :

Color tone :

Color Space :

NIKON D5100
f/5.3
$1 / 60 \mathrm{sec}$
$+0.7 \mathrm{steps}$
$40 \mathrm{~mm}$
4.8
Centre avg.
$1 / 200 \mathrm{Sec}$.
F5.6

$+2 / 3$
2000
$55.0 \mathrm{~mm}$
$4928 \mathrm{x} 3264$
Manual
(Normal(-))
Normal
Normal
Normal
Normal
Normal
sRGB




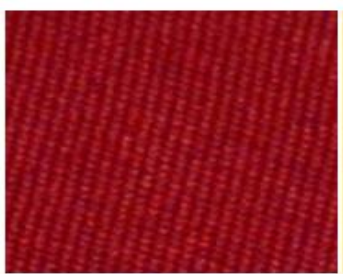

11

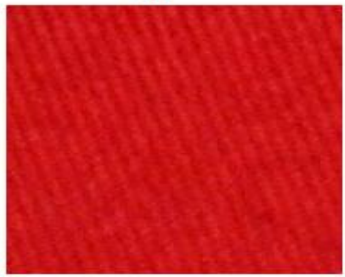

15

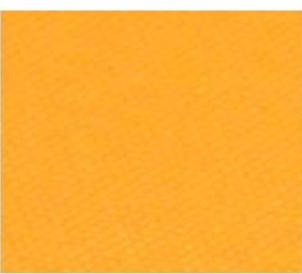

12

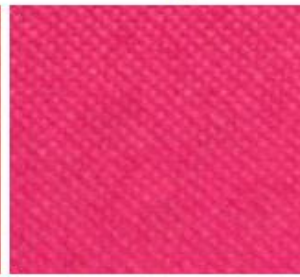

16

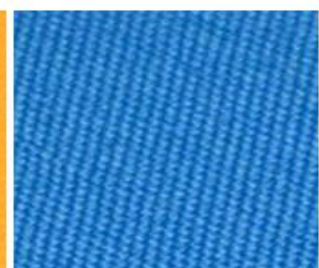

13

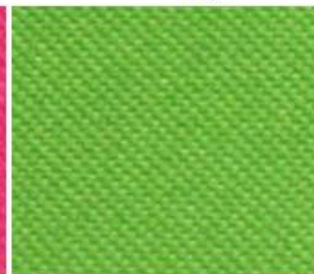

17

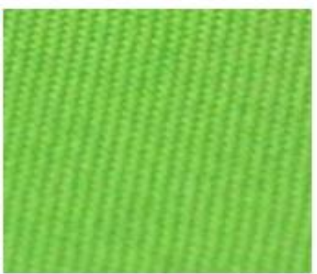

14

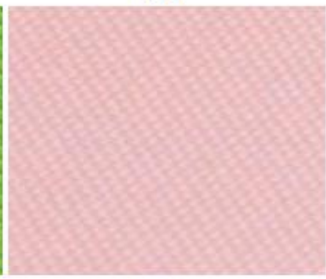

18

Fig.3. Eight own created texture images.

Table 2. Fractal Dimension of Eight own Created Texture Images

\begin{tabular}{|c|c|c|}
\hline Image Name & Proposed & Box Merging \\
\hline I1 & 3.4712 & 2.8379 \\
\hline I2 & 2.6839 & 2.4818 \\
\hline I3 & 3.8591 & 3.1798 \\
\hline I4 & 3.4151 & 2.6646 \\
\hline I5 & 3.2723 & 2.8894 \\
\hline I6 & 3.3925 & 2.7117 \\
\hline I7 & 3.5295 & 2.8153 \\
\hline I8 & 3.1442 & 2.6667 \\
\hline
\end{tabular}

\section{Experiment on Known Fractal dimension Images}

In this section, we are using a set of 4 smooth texture images with varied color, which are represented in "Fig. 4," .Their corresponding FD are listed on Table 3, from this experimental analysis, we have seen that our proposed method also able to capture smoothness of color images.

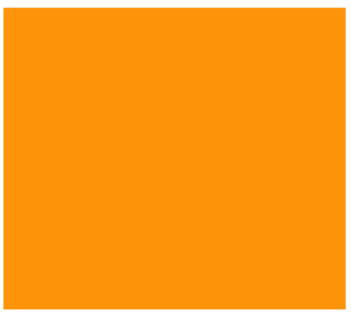

a

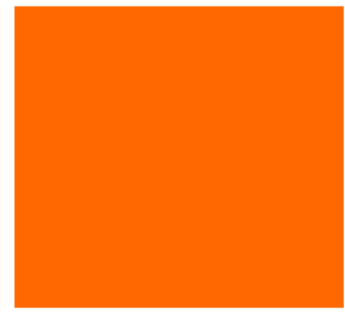

b

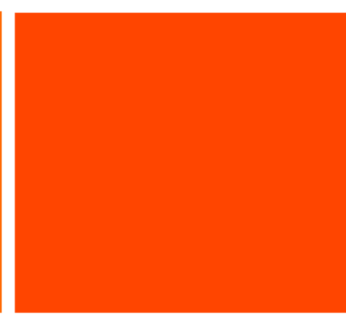

C

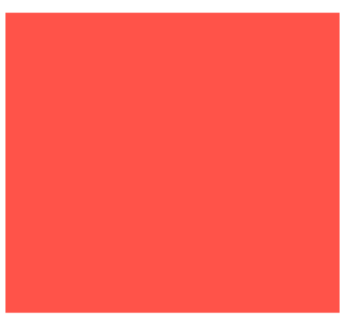

d

Fig.4. Four known fractal dimension smoothed images.

Table 3. Fractal Dimension of Four Known Fractal Dimension Smoothed Images.

\begin{tabular}{|c|c|c|}
\hline Image Name & Proposed & Box merging \\
\hline $\mathrm{a}$ & 2 & 2 \\
\hline $\mathrm{b}$ & 2 & 2 \\
\hline $\mathrm{c}$ & 2 & 2 \\
\hline $\mathrm{d}$ & 2 & 2 \\
\hline
\end{tabular}

\section{E. Experiment on Synthetic Images}

In this experimental setup, Twelve synthesize images are generated with maximum gray level of 255 of each red, green and blue channel, which are represented in "Fig. 4,". In this process, each image ware generated from original image by incrementing each intensity point (by formula $2 \times(k-2)$, where $\mathrm{k}$ lies from 1 to 12 ) in such a way that at the $k^{\text {th }}$ image of $12^{\text {th }}$ image, the maximum gray level should not exceed 255 for each channel, therefore alternatively we can say that all the intensity 
surface are only shifted to up some gray levels from the original one in $\mathrm{z}$ direction. Theoretically it was clear that, if we either increasing or decreasing by a constant value, then the FD should stay same as original one because theoretically both have equal degree of roughness. The estimated FD are listed in Table 4 and graph are represented in "Fig. 5,", from the result, we have seen that our proposed method indicates same roughness for all the images but box merging method does not provides wider range of FD.
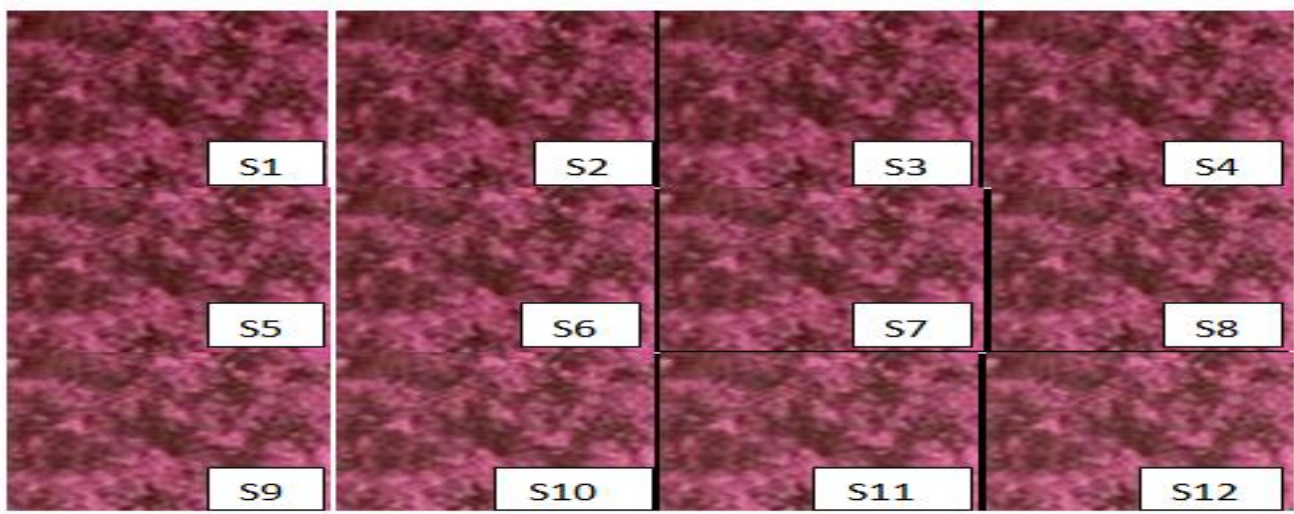

Fig.5. Twelve synthetic generated images

Table 4. Computational FD of Twelve Synthetic Images.

\begin{tabular}{|c|c|c|}
\hline Image Name & Proposed & Box merging(BM) \\
\hline S1 & 3.8735 & 3.5007 \\
\hline S2 & 3.8735 & 2.8651 \\
\hline S3 & 3.8735 & 2.8797 \\
\hline S4 & 3.8735 & 2.8571 \\
\hline S5 & 3.8735 & 2.8628 \\
\hline S6 & 3.8735 & 2.8039 \\
\hline S7 & 3.8735 & 2.7948 \\
\hline S8 & 3.8735 & 2.7250 \\
\hline S9 & 3.8735 & 2.6275 \\
\hline S10 & 3.8735 & 2.6228 \\
\hline S11 & 3.8735 & 2.6180 \\
\hline S12 & 3.8735 & 2.6229 \\
\hline
\end{tabular}

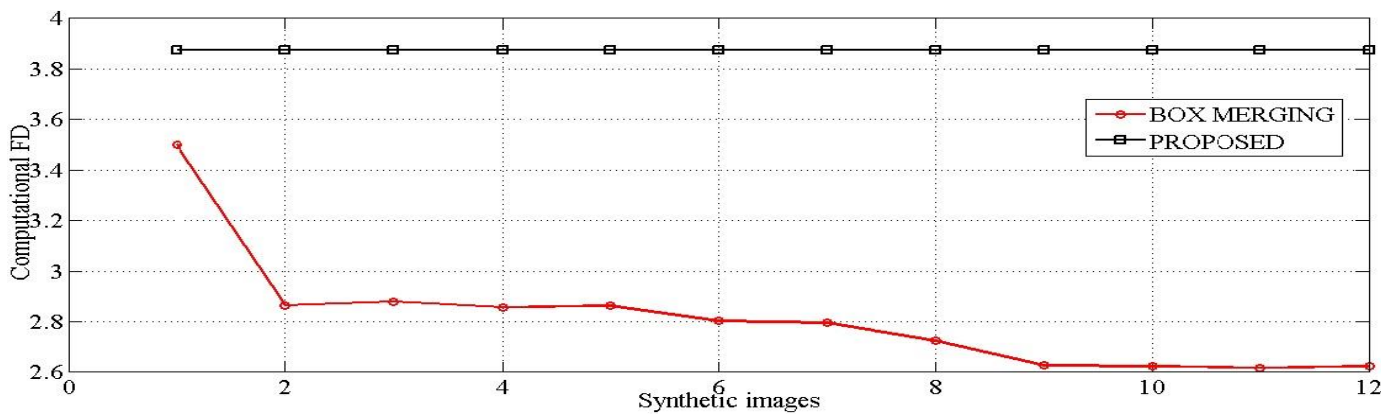

Fig.6. Graph of Twelve synthetic generated images

\section{CONCLUSION}

In this paper, we presented a color extension of the improved differential box-counting algorithm, which was widely used to compute the fractal dimension of gray scale images. Theoretically, for a color image is a 5-D fractal. As color image consists of more intensity levels than the grayscale image, the fractal dimension method suggested by us indicates an estimation of the much better accuracy of roughness. The fractal dimension of more than 3.0 we measure indicates the fact that our algorithm it is able to capture the complexity of a color image.

\section{REFERENCES}

[1] B. B. Mandelbrot, Fractal Geometry of Nature. San Francisco: Freeman, 1982.

[2] A. P. Pentland, "Fractal-based description of natural scenes," IEEE Transaction on Pattern Analysis and Machine Intelligence, vol. 6, pp. 661-674, 1984.

[3] H. O. Peitgen, H. Jurgens, D. Saupe, Chaos and Fractals: New Frontiers of Science, first ed, Springer, Berlin, 1992. 
[4] B. B. Chaudhuri, N Sarker, "Texture segmentation using fractal dimension," IEEE Transaction on Pattern Analysis and Machine Intelligence, vol. 17, pp. 72-77, 1995.

[5] S. Liu, S. Chang, "Dimension estimation of discrete-time fractional Brownian motion with applications to image texture classification," IEEE Transaction on Image Processing, vol. 6, vol. 1176-1184, 1997.

[6] T. Ida, Y. Sambonsugi, "Image segmentation and contour detection using fractal coding," IEEE Trans. Circuits System Video Technology, vol. 8, pp. 968-977, 1998.

[7] G. Neil, K. M. Curtis, "Shape recognition using fractal dimension," Pattern Recognition, vol. 30, pp. 1957-1969, 1997.

[8] K. H. Lin, K. M. Lam, W. C. Siu, "Locating the eye in human face images using fractal dimensions," IEEE Proceedings on Vision, Image and Signal Processing, vol. 148, pp. 413-421, 2001.

[9] P. Asvestas, G. K. Matsopoulos, K. S. Nikita, "A power differentiation method of fractal dimension estimation for 2-D signals," Journal of Visual Communication and Image Representation, vol. 9, pp. 392-400, 1998.

[10] A. K. Bisoi, J. Mishra, "On calculation of fractal dimension of images," Pattern Recognition Letters, vol. 22, pp. 631-637, 2011

[11] J. J. Gangepain, C. R. Carmes, "Fractal approach to two dimensional and three dimensional surface roughnesses," Wear, vol. 109, pp. 119-126, 1986.

[12] J. M. Keller, R. M. Crownover, S. Chen, "Texture description and segmentation through fractal geometry," Comp. Vision Graph. Image Processing, vol. 45, pp. 150160, 1989.

[13] N. Sarker, B. B. Chaudhuri, "An efficient differential boxcounting approach to compute fractal dimension of image," IEEE Trans. Systems, Man, and Cybernetics, vol. 24, pp. 115-120, 1994.

[14] X. Wenlu, X. Weixin, "Fractal-based analysis of time series data and features extraction," Signal Processing, vol. 13, pp. 98-104, 1997.

[15] L. Yu, D. Zhang, K. Wang, W. Yang, "Coarse iris classification using box-counting to estimate fractal dimensions," Pattern Recognition, vol. 38, pp. 1791-1798, 2005.

[16] J. Li, Q. Du, C. Sun, “An improved box-counting method for image fractal dimension estimation," Pattern Recognition, vol. 42, pp. 2460-2469, 2009.

[17] Y. Liu, L. Chen, H. Wang, L. Jiang, Y. Zhang, J. Zhao, D. Wang, Y. Zhao, Y. Song, "An improved differential boxcounting method to estimate fractal dimensions of graylevel images," Journal of Visual Communication and Image Representation, vol. 25, pp. 1102-1111, 2014.
[18] P. Brodatz, Texture: A Photographic Album for Artists and Designers, New York, 1966.

[19] M. Ivanovici, N. Richard, "Fractal dimension of colour fractal images," IEEE Transaction on Image Processing, vol. 20, pp. 227-235, 2011.

[20] N. S. Nikolaidis, I. N. Nikolaidis, C.C. Tsouros, "A Variation of the Box-Counting Algorithm Applied to Colour Images," arXiv preprint arXiv, pp. 1107-2336, 2011.

[21] X. Zhao, X. Wang, "Fractal dimension estimation of RGB color images using maximum color distance," Fractals, vol. 24, pp. 1650040-1650055, 2016.

\section{Authors' Profiles}

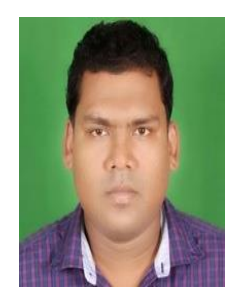

Soumya Ranjan Nayak was born in odisha in 1984. He received his B.Tech and M.Tech degree in computer science and engineering from Biju Patnaik University of Technology, Odisha, India in 2009 and 2012, respectively. Currently, he is working as research scholar in college of engineering and technology, odisha. His research interest includes image analysis by means of fractal geometry, color and texture features.

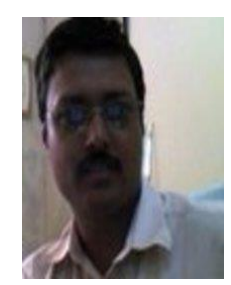

Jibitesh Mishra was born in odisha in 1968 . He received his MCA degree from the Utkal University, Odisha India, and the Ph.D. degrees from the Utkal University, Odisha India, in 2001. Currently, he is a Associate Professor and Head, Department of Computer Science and Application, College of Engineering and Technology, Bhubaneswar, a constituent college of Biju Patnaik University of Technology, Odisha. He has more than 16 years of teaching experience in various universities throughout the world. He has authored four books of repute. His research interests are fractal graphics.

How to cite this paper: Soumya R. Nayak, Jibitesh Mishra,"On Calculation of Fractal Dimension of Color Images", International Journal of Image, Graphics and Signal Processing(IJIGSP), Vol.9, No.3, pp.33-40, 2017.DOI: 10.5815/ijigsp.2017.03.04 